\title{
Das österreichisch-ungarische Grenzgebiet Entwicklungschancen und -probleme im Zuge der Grenzöffnung
}

\section{Einleitung}

\section{Grenze und geographische Grenzforschung}

Staatsgrenzen sind wesentliche Elemente der Kulturlandschaft, genauer der politischen Systeme im Raum, mit diametraler Funktion. Als Barrieren oder Schranken begrenzen sie nicht nur den staatlichen Wirkungsbereich und sein Territorium, sondern ebenso die wirtschaftlichen, sozialen und kulturellen Beziehungen zwischen der Bevölkerung beiderseits der Grenze. Mit steigender Durchlässigkeit nimmt dagegen ihre verbindende Funktion zu. So finden räumliche Prozesse auch jenseits der Staatsgrenze eine Fortsetzung oder erfahren Umstrukturierungen. Das Ausmaß der Geschlossenheit bzw. Durchlässigkeit einer Grenze steht wiederum in direkter Abhängigkeit zu den herrschenden politisch-ökonomischen und gesellschaftlichen Systemen in den durch die Grenze getrennten Staaten.

Im zeitlichen Prozeß unterliegen politische Grenzen Funktionsänderungen, wie das Beispiel der europäischen Staatsgrenzen verdeutlicht. Mit dem Wandel der Funktion einer Grenze erfahren zumeist auch die Grenzgebiete eine Umbewertung. So können peripher gelegene, wirtschaftlich benachteiligte Gebiete durch die Auflösung politischer und wirtschaftlicher Schranken in eine zentralere und damit günstigere Position innerhalb kooperierender Staaten rücken. ${ }^{1}$ Umgekehrt können Grenzregionen durch die Verstärkung der trennenden Funktion einer Grenze eine qualitative Abwertung erfahren und in ihrer wirtschaftlichen Entwicklung behindert werden.

Betrachtet man den Stand der grenzgeographischen Forschungen, so ist festzustellen, daß die EU-«Binnengrenzen» bereits ein vielfältig untersuchtes geographisches Objekt ${ }^{2}$ darstellen. Dies steht in engem Zusammenhang mit dem Bedeutungszuwachs der Grenzregionen für den gesamteuropäischen Integrationsprozeß. $\mathrm{Zu}$ einer Belebung der Grenzraumforschung im osteuropäischen Raum kam es jedoch erst durch die dortigen politischen Umbrüche und grundlegenden Umwälzungen. Im Zuge der sogenannten «friedlichen Revolution» rückten die Grenzen zwischen West und Ost wieder in den Blickpunkt des politischen, öffentlichen und wissenschaftlichen Interesses. ${ }^{3}$

Der Fall des Eisernen Vorhangs, in kausaler Verbindung mit dem Zusammenbruch des sozialistischen Wirtschafts- und Gesellschaftssystems, ist ein weiteres Beispiel für die Funktionsveränderung von Grenzen und die Abhängigkeit ihrer Wertigkeit von den Systemen der aneinandergrenzenden Staaten.

\section{Ziel der Studie}

Der vorliegende Beitrag befaßt sich mit den aktuellen Auswirkungen der Ostöffnung auf die Gebiete beiderseits der österreichisch-ungarischen Staatsgrenze (Abb. 1) und den daraus resultierenden Entwicklungschancen und -problemen. Im Mittelpunkt des Interesses steht dabei die Frage nach der Funktionsänderung der Grenze, d. h. dem tatsächlichen Grad ihrer Durchlässigkeit und den Auswirkungen der Öffnung einer «geschlossenen» Grenze auf das Verhalten der im Grenzraum ansässigen Bevölkerung und der wirtschaftlichen Entscheidungsträger und damit letztlich auf die Entwicklung der peripheren Grenzgebiete. Auch werden Faktorenkomplexe ermittelt, die das Ausmaß und die Intensität der grenzübergreifenden Beziehungen beeinflussen und das Zusammenwachsen der Grenzgebiete hemmen bzw. fördern.

\section{Relevanz der Studiengebiete für die Grenzraumforschung}

Die österreichisch-ungarische Grenze und ihre peripheren Grenzgebiete sind im gegebenen Kontext insofern ein besonders geeignetes geographisches Forschungsobjekt, als ihre Lage und Funktion in einem historisch gesehen kurzen Zeitraum mehrfach grundlegende Veränderungen erfuhr. Durch den Friedensvertrag von Trianon 1920 wurde Österreich ein Teil des ungarischen Staatsgebietes zugesprochen, aus dem als neuntes österreichisches Bundesland das Burgenland hervorging. Trotz der Grenzziehung blieb der so geteilte Raum weitgehend als einheitlicher Lebens- und Wirtschaftsraum bestehen («offene Grenze»). Erst die als Folge des Zweiten Weltkrieges entstandenen völlig konträren politischen und gesellschaftlichen Systeme beiderseits der Grenze führten zu einer divergierenden räumlichen

Andrea Kampschulte, Dr., Departement Geographie, Uni Basel, Klingelbergstraße 16, 4056 Basel 


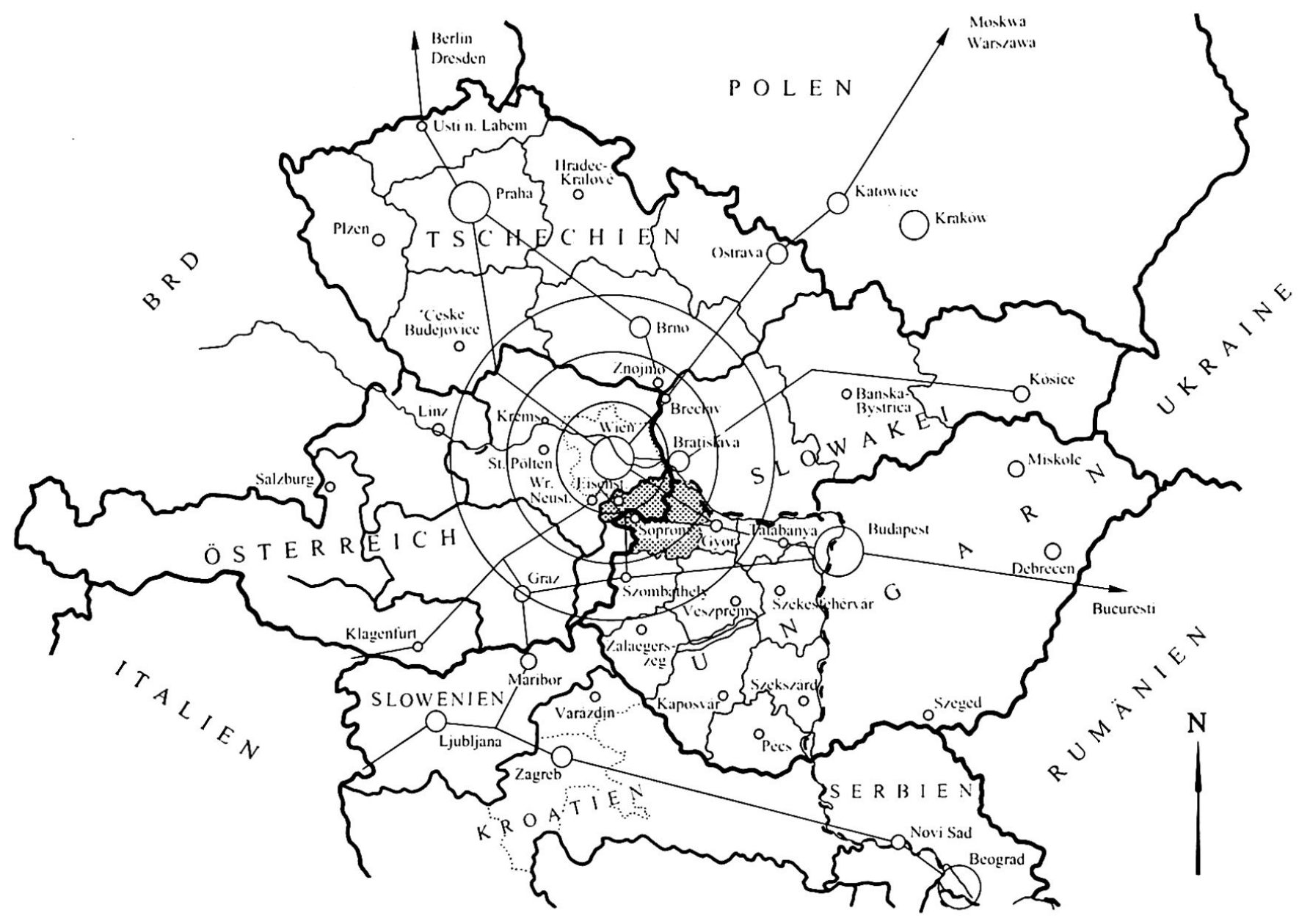

Abb. 1 Großräumige Lage des österreichisch-ungarischen Untersuchungsgebietes

Quelle: Eigener Entwurf, verändert nach Planungsgemeinschaft Ost, Berichte und Veröffentlichungen 1/1991, S. 11.

Entwicklung und zur vollständigen Abschottung der Staaten gegeneinander, die ihren stärksten Ausdruck 1948 in der Errichtung des Eisernen Vorhangs fand («geschlossene Grenze»). Einem allmählichen Abbau der trennenden Funktion der Grenze seit Beginn der 80er Jahre folgte mit der weitgehenden Reisefreiheit der ungarischen Bürger ab dem 1. Januar 1988 und dem endgültigen Fall der Grenzbarrikaden mit der Deklamation der Ungarischen Republik am 23. Oktober 1989 erneut eine Phase der «offenen Grenze» und damit neue Möglichkeiten zur Wiederbelebung und Intensivierung der grenzüberschreitenden Beziehungen.

\section{Die grenzüberschreitenden Verflechtungen der Bevölkerung im österreichisch-ungarischen Grenzraum}

\section{Vorbemerkungen zur empirischen Analyse}

Die folgenden Ausführungen geben zunächst die Ergebnisse einer partiellen Regionalanalyse und im An- schluß die Resultate umfangreicher empirischer Untersuchungen zu den grenzüberschreitenden Verflechtungen der Bevölkerung, gemessen am Verhalten der einzelnen Haushalte, und den zwischenbetrieblichen Kooperationen wieder. Haushalte und Betriebe wurden als Untersuchungseinheiten ausgewählt, da sich anhand beider Gruppen der Wandel im Zuge der Grenzöffnung relativ rasch, deutlich und quantitativ meßbar erfassen läßt. ${ }^{4}$ Als Untersuchungsgebiet wurden das nördliche Burgenland und in Abhängigkeit der Entfernung zur Grenze vier der fünf ehemaligen Stadt-Umland-Zonen im Komitat Györ-Moson-Sopron (Abb. 2) ausgewählt. Insgesamt konnten im Zeitraum 1992/93 mittels schriftlicher Befragungen über Schulklassen 893 Haushalte im österreichischen und 693 Haushalte im ungarischen Grenzgebiet erfaßt werden. In die Stichprobe der als Postwurfumfrage durchgeführten Betriebsbefragung wurden, im Hinblick auf die notwendigen Voraussetzungen für grenzüberschreitende Kooperationen, nur Betriebe ab fünf unselbständig Beschäftigten aufgenommen. Untersucht wurden insgesamt 55 nordburgenländische und 38 westungarische Betriebe. 


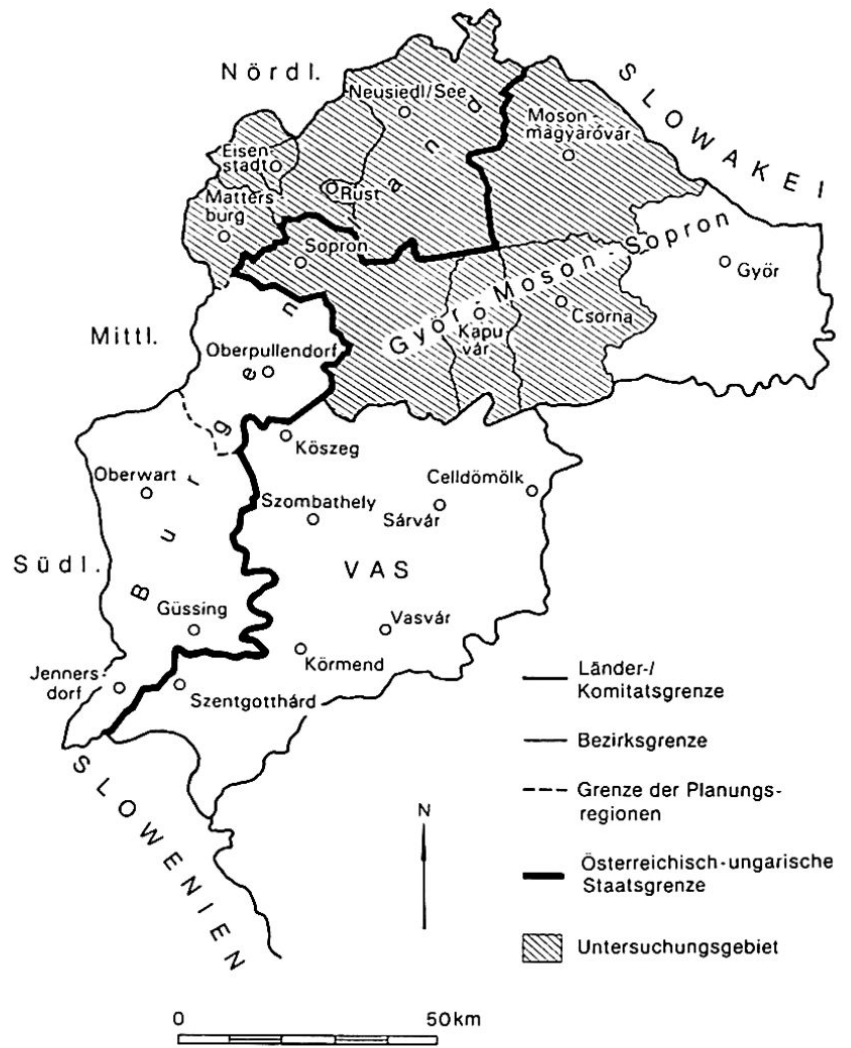

Abb. 2 Das österreichisch-ungarische Untersuchungsgebiet Quelle: Eigener Entwurf.

\section{Gemeinsamkeiten und Unterschiede der Grenzgebiete - Ergebnisse der partiellen Regionalanalyse}

Zwischen den Grenzregionen bestehen zahlreiche Gemeinsamkeiten, aber auch wesentliche strukturelle Unterschiede. Diese sind besonders im Hinblick auf Richtung und Intensität der grenzüberschreitenden Interaktionen und Kooperationen von entscheidender Relevanz. Zu den Gemeinsamkeiten zählen die naturräumlichen Gegebenheiten und die historische Entwicklung. Das gemeinsame historische Erbe stellt folglich eine wesentliche Grundlage für die Wiederherstellung bzw. Intensivierung der kulturellen und sozialen Beziehungen im Grenzraum dar. Die Tatsache, daß beide Gebiete traditionell zu einem Wirtschaftsund Kulturraum zählten und enge politische, soziale und ökonomische Verflechtungen aufwiesen, eröffnet grundlegende Anknüpfungsmöglichkeiten.

Bedeutende Unterschiede weisen die Bevölkerungs-, Siedlungs- und Wirtschaftsstruktur in beiden Teilgebieten auf. Für die untersuchten grenzüberschreitenden Interaktionen und Kooperationen sind dabei vor allem das Fehlen größerer Städte im nördlichen Burgenland infolge der Grenzziehung von 1921 sowie die abweichenden
Betriebsgrößen- und industriellen Branchenstrukturen beiderseits der Grenze von Bedeutung. Letztere sind primär Folge der divergierenden Wirtschafts- und Gesellschaftssysteme in beiden Staaten nach dem Zweiten Weltkrieg.

Als wesentliche Grenzgefälle zwischen den Untersuchungsgebieten können die Unterschiede in der Höhe der Steuern und Zölle identifiziert werden, die sich aus den politischen Trennwirkungen der Grenze ergeben. Zum anderen bestehen Differenzen hinsichtlich des Wohlstandes, der Kaufkraft und der Preise und Löhne, die auf den unterschiedlichen wirtschaftlichen Entwicklungsstand zurückzuführen sind. Darüber hinaus sind auch die Kenntnisse der jeweiligen Nachbarsprache verschieden stark verbreitet.

Unabhängig von den interregionalen Differenzen unterscheiden sich beide Grenzgebiete schließlich durch ihren Stellenwert im gesamtstaatlichen Gefüge. Während das nördliche Burgenland innerhalb Österreichs zu den entwicklungsschwachen peripheren Gebieten zu rechnen ist, zählt das Komitat Györ-MosonSopron seit der Grenzöffnung zu den begünstigten Räumen Ungarns. Die Tatsache, daß in beiden Ländern ein ausgeprägtes West-Ost-Gefälle vorliegt, bedeutet zugleich, daß zwischen den Grenzgebieten die relativ geringsten Entwicklungsunterschiede und damit die vergleichsweise günstigsten Voraussetzungen für eine grenzübergreifende Zusammenarbeit gegeben sind.

\section{Ergebnisse der Haushaltsbefragung}

Intensität der grenzüberschreitenden Beziehungen

Als ein wesentlicher Indikator für den Wandel der Durchlässigkeit der österreichisch-ungarischen Staatsgrenze kann die Intensität der grenzüberschreitenden Beziehungen herangezogen werden. Laut den empirischen Untersuchungen sind diese seit der Ostöffnung deutlich angestiegen. Dies wird durch die Anzahl der erstmaligen Grenzübertritte der befragten Haushalte, die ihre Maxima in beiden Grenzgebieten zwischen 1988 und 1991 - also zeitgleich mit der Liberalisierung in Ungarn - erreichen, nachgewiesen. Im zeitlichen Verlauf spiegeln die Anteile der erstmaligen Einreise in das Nachbarland zudem deutlich die einzelnen Etappen der Grenzpolitik zwischen Österreich und Ungarn im dargestellten Zeitraum wider (Abb.3).

Nach Verschärfung der Grenzsperren und Erschwerung der Einreise infolge des Volksaufstandes 1956 haben zwischen 1960 und 1980 maximal zwei Prozent der Befragten pro Jahr die Grenze nach Ungarn erstmals überschritten. Erst nach der Rücknahme des Visumzwangs für österreichische Staatsbürger 1978 wird 1980 ein erster Höhepunkt der Grenzübertritte erreicht. In diesem Jahr fahren $11,8 \%$ der Befragten zum ersten Mal nach Ungarn. In der Folgezeit sind es pro Jahr durchschnittlich fünf Prozent, bis im Zeitraum zwischen 1989 und 1991 mit Anteilen zwischen 12,2\% und 14,8\% weitere 


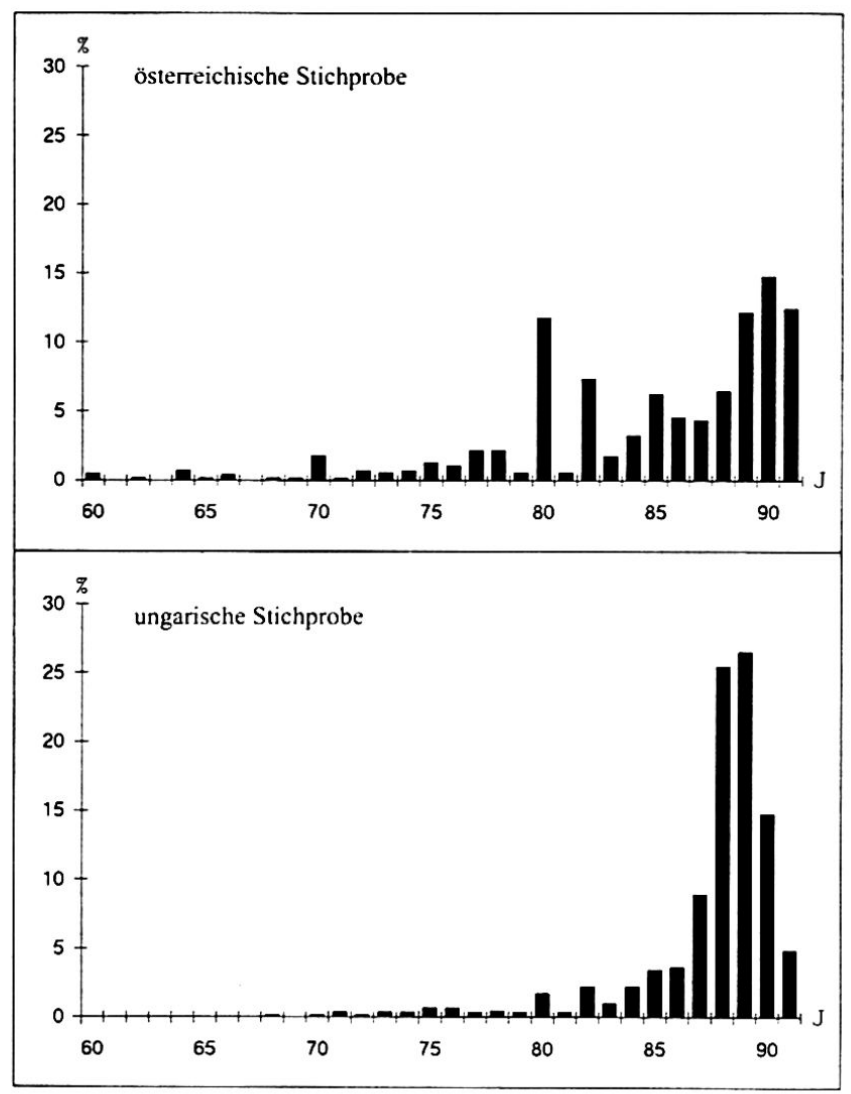

Abb. 3 Erstmalige Grenzübertritte nach Jahreszahl (Nördliches Burgenland $n=725$; Westungarn $n=628$ )

Quelle: Eigene Erhebungen.

Maxima bei der erstmaligen Einreise auftreten. Diese fallen zeitlich mit dem endgültigen Fall des Eisernen Vorhangs zusammen.

Auf ungarischer Seite sind den erschwerten Bedingungen für eine Ausreise entsprechend bis 1970 kaum Grenzübertritte der Befragten nach Österreich zu verzeichnen. Noch bis 1980 reisen weniger als ein Prozent der Haushalte pro Jahr erstmals nach Österreich. Mit dem allmählichen Abbau der trennenden Funktion der Grenze seit Beginn der 80er Jahre nimmt der Anteil der betreffenden Haushalte auf durchschnittlich drei Prozent zu. Aber erst mit der Ausstellung des Weltreisepasses seit dem 1.1.1988 steigen die Einreisen ins benachbarte Österreich sprunghaft an. 1988 nutzen $25,5 \%$ der Befragten diese Möglichkeit. Im Jahr des endgültigen Falls der Grenzbarrikaden und der Deklamation der Ungarischen Republik am 23.10.1989 erreicht die Zahl der erstmaligen Grenzübertritte mit 26,6\% der Befragten dann auch im ungarischen Untersuchungsgebiet ihr Maximum.

Die als Maß für die Intensität der grenzüberschreitenden Beziehungen ermittelten Häufigkeitsindizes ${ }^{5}$ zeigen, daß die westungarischen Haushalte im Durchschnitt deutlich häufiger nach Österreich fahren als die Nordburgenländer umgekehrt nach Ungarn. Die grundsätzliche Bedeutung der Verflechtungen mit dem benachbar- ten Grenzgebiet läßt sich daraus ablesen, daß nur jeder fünfte österreichische und jeder zehnte ungarische Haushalt noch nie von der Möglichkeit des Grenzübertritts Gebrauch gemacht hat. Die Mehrzahl der Befragten in beiden Untersuchungsgebieten besucht das jeweilige Nachbarland mehrmals pro Jahr.

\section{Einflußgrößen für die Häufigkeit der Grenzübertritte}

Ausmaß und Intensität der grenzüberschreitenden Beziehungen sind von einer Vielzahl von (haushaltsspezifischen) Merkmalen abhängig. Zu den Einflußgrößen, die die Häufigkeit der Grenzübertritte in beiden Untersuchungsgebieten maßgeblich bestimmen, zählen die Kenntnis der Nachbarsprache (Abb. 4), die persönlichen Bindungen über die Grenze und die Möglichkeit zur Kopplung verschiedener Aktivitäten im benachbarten Ausland. Während im ungarischen Untersuchungsgebiet die Befragten mit beruflichen Kontakten zu Österreichern den höchsten Häufigkeitsindex erreichen $\left(H_{i} 5,5\right)$, sind dies im nördlichen Burgenland diejenigen, die über ungarische Sprachkenntnisse verfügen $\left(\mathrm{H}_{\mathrm{i}}\right.$ 3,3). Den zwischen den Grenzgebieten bestehenden Mobilitäts- und Einkommensunterschieden entsprechend, wird die Intensität der Beziehungen über die Grenze auf ungarischer Seite zudem wesentlich durch die Distanz zum benachbarten Grenzraum, den PKWBesitz (Abb. 5), die Haushaltsgröße und den Sozialstatus beeinflußt. Darüber hinaus variiert die Einreisehäu-
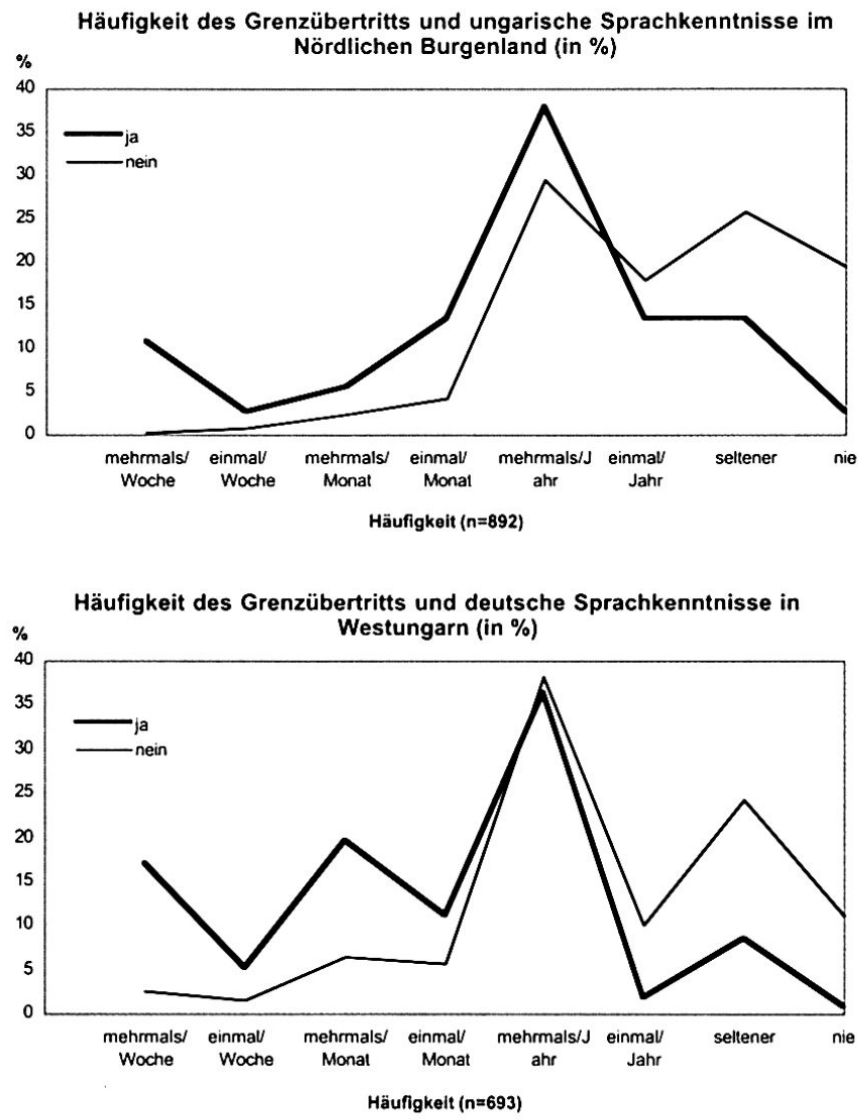

Abb. 4 Häufigkeit des Grenzübertritts und Sprachkenntnisse (in \%) Quelle: Eigene Erhebungen. 


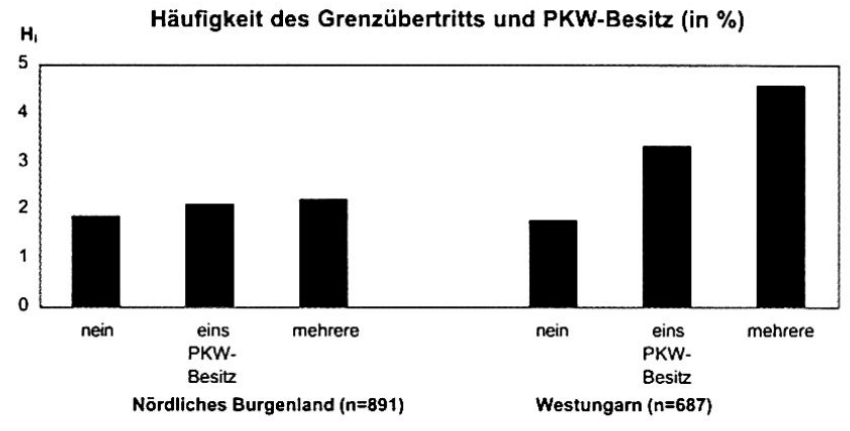

Abb. 5 Häufigkeit des Grenzübertritts und PKW-Besitz Quelle: Eigene Erhebungen.

figkeit in das jeweilige Nachbarland in Abhängigkeit von den Fahrtgründen sowie deren Kopplungseignung.

\begin{abstract}
Fahrtmotive
Unter den verschiedenen Fahrtmotiven kommt dem Einkauf in der Gesamtheit der grenzüberschreitenden Verflechtungen in beiden Untersuchungsgebieten, insbesondere aber auf ungarischer Seite, der mit Abstand größte Stellenwert zu. Hier erfolgen $86 \%$ der Grenzübertritte zu Versorgungszwecken, wenngleich diese nicht stets den alleinigen oder primären Fahrtgrund darstellen. Während im direkten Vergleich den Grenzübertritten der Nordburgenländer stärker freizeitorientierte Motive (Stadtbummel, Ausflüge, Gaststättenbesuche) zugrunde liegen, spielen bei den ungarischen Haushalten berufliche Gründe eine größere Rolle. Die Unterschiede im Stellenwert der einzelnen Fahrtmotive spiegeln vor allem den auf ungarischer Seite zum Zeitpunkt der Untersuchung bestehenden Nachholbedarf im konsumtiven Bereich einerseits und die besseren Verdienstmöglichkeiten in Österreich bei gleichzeitig steigender Arbeitslosigkeit im ungarischen Grenzgebiet andererseits wider.
\end{abstract}

\section{Grenzüberschreitende Einkaufsbeziehungen}

Hinsichtlich ihrer Intensität, Art und Reichweite weichen die Einkaufsbeziehungen zwischen den Grenzgebieten grundlegend voneinander ab. So nutzen auf ungarischer Seite nicht nur eine größere Anzahl von Haushalten die Möglichkeit des grenzübergreifenden Einkaufs, sondern diese fahren auch durchschnittlich häufiger zu Versorgungszwecken nach Österreich. Die Entscheidung über die Inanspruchnahme ausländischer Versorgerorte wird dabei in beiden Grenzgebieten in erster Linie durch die Merkmale Wohndauer, Haushaltsgröße, Freizeitvolumen und PKW-Verfügbarkeit beeinflußt. Für die Intensität der Einkaufsbeziehungen ist außerdem die Bedarfshäufigkeit der überwiegend nachgefragten Waren von Relevanz.

Unterschiede in der Art der Versorgungsbeziehungen bestehen sowohl hinsichtlich der bevorzugt erworbenen Artikel (Lebensmittel, Drogerie-, Elektro- und Haus- haltwaren in Österreich / Lebensmittel und Bekleidungsartikel in Ungarn) als auch in bezug auf die den Einkäufen zugrunde liegenden Motivationen. So beruht die Bedarfsdeckung in Ungarn primär auf dem Preisgefälle, die in Österreich hingegen auf der Angebotsvielfalt und Qualität der Produkte, wenngleich auch hier Preisunterschiede von Relevanz sind. Da insgesamt die Mehrzahl der Einkäufe im jeweiligen Nachbarland aufgrund von Preis- oder Qualitätsdifferenzen erfolgt, liegen in beiden Grenzgebieten jedoch überwiegend keine echten zentralörtlichen Beziehungen zu den ausländischen Zentren vor.

Differenzen in der Reichweite der grenzübergreifenden Einkaufsorientierungen sind vor allem in den spezifischen zentralörtlichen Systemen beiderseits der Grenze begründet. Dem von der überwiegenden Mehrheit der burgenländischen Haushalte in Anspruch genommenen, grenznah gelegenen Sopron steht im nördlichen Burgenland kein entsprechend attraktiver Versorgerort oberer Stufe gegenüber. Die ungarischen Konsumenten sind daher zum Großteil auf die zwar weiter entfernten, dafür aber besser ausgestatteten Oberzentren Wien und Wiener Neustadt ausgerichtet. Des weiteren weisen die westungarischen Haushalte durchschnittlich größere Aktionsreichweiten und deutlich vielfältigere grenzüberschreitende Orientierungen auf.

Im Gesamtzusammenhang der binnenräumlichen Versorgungsbeziehungen kommt der Bedarfsdeckung im Nachbarland in beiden Untersuchungsgebieten jedoch ein vergleichsweise untergeordneter Stellenwert zu. Lediglich in einigen meist grenznah gelegenen Gemeinden werden die ausländischen Zentren regelmäßig und alternativ zu inländischen Versorgerorten aufgesucht. Sie tragen in diesen Gemeinden gleichzeitig zur Verbesserung der Versorgungssituation bei.

\section{Affektive Einstellung zum ausländischen Nachbarn}

Als ein weiterer, die «Qualität» der grenzüberschreitenden Beziehungen determinierender Faktor wurde die affektive Einstellung zum Nachbarland und seiner Bevölkerung angenommen. Diesbezüglich zeigte sich, daß sowohl im nördlichen Burgenland als auch in Westungarn die Mehrheit der Haushalte eine positive Einstellung zum ausländischen Nachbarn und zur Grenzöffnung aufweist. Im Hinblick auf die zukünftige Entwicklung des Grenzraumes sind die ungarischen Haushalte jedoch deutlich optimistischer. Unter den Gründen für eine positive Beurteilung der Ostöffnung stehen in beiden Untersuchungsgebieten die persönliche Freiheit und wirtschaftliche Vorteile an vorderster Stelle. Beeinflußt wird die affektive Einstellung primär durch die persönlichen grenzüberschreitenden Kontakte, die Sprachkenntnisse und die Sozialgruppenzugehörigkeit. Ein Zusammenhang zwischen der Intensität der grenzübergreifenden Verflechtungen und der Haltung zum Nachbarn läßt sich hingegen nur für die burgenländischen Haushalte nachweisen. Nicht eruiert werden konnte hierbei jedoch das Ausmaß der gegenseitigen Beeinflussung beider Merkmale. 
Als wesentliche Faktoren für ein zukünftiges «Zusammenwachsen» der Grenzgebiete können gemäss den Untersuchungen somit die persönlichen Kontakte zur benachbarten Grenzbevölkerung und die Kenntnis der Nachbarsprache sowie die hiermit eng verknüpften Informationsmöglichkeiten angesehen werden.

\section{Wirtschaftliche Perspektiven und Kooperationen der Betriebe im österreichisch-ungarischen Grenzraum $^{6}$ - Ergebnisse der Betriebsbefragung}

Im Mittelpunkt der empirischen Untersuchungen standen hier die Reaktionen der Betriebe auf die Liberalisierung in Ungarn, ferner Umfang, Formen und Motive der wirtschaftlichen Zusammenarbeit zwischen den Grenzgebieten vor und seit der Grenzöffnung sowie die in diesem Zusammenhang bereits gewonnenen Erfahrungen.

\section{Einschätzung der regional- und betriebs- wirtschaftlichen Situation im Zuge der Ostöffnung}

Insgesamt sind die zwischenbetrieblichen grenzüberschreitenden Kontakte und Kooperationen im Untersuchungsgebiet seit der Ostöffnung beträchtlich ausgebaut worden. Dabei steht die Mehrheit der befragten Betriebe der Grenzöffnung und den damit verbundenen Auswirkungen überwiegend positiv gegenüber. Hieraus resultierende Vorteile werden jedoch vor allem für die regionale Entwicklung infolge einer allgemeinen Belebung der Wirtschaft ausgemacht, während die betrieblichen Perspektiven (Tab. 1) in beiden Grenzgebieten sehr unterschiedlich beurteilt werden. Der vergleichsweise stark ausgeprägte Pessimismus der westungarischen Betriebe ist dabei in erster Linie auf die Anpassungskrisen zurückzuführen, die durch den Wechsel des Wirtschaftssystems hervorgerufen werden.

Tab.1: Einschätzung der wirtschaftlichen Situation der erfaßten Betriebe (in \%)

\begin{tabular}{|c|c|c|c|c|}
\hline \multirow{3}{*}{$\begin{array}{l}\text { Einschätzung der } \\
\text { wirtschaftlichen } \\
\text { Situation }\end{array}$} & \multicolumn{4}{|c|}{ Betriebe im } \\
\hline & \multicolumn{2}{|c|}{$\begin{array}{l}\text { österreichischen } \\
\text { Untersuchungsgebiet ( } n=51 / 55)\end{array}$} & \multicolumn{2}{|c|}{$\begin{array}{c}\text { ungarischen } \\
\text { Untersuchungsgebiet }(n=38)\end{array}$} \\
\hline & gegenwärtig & zukünttig & gegenwärtig & zukünftig \\
\hline sehr gut & 19.6 & 14.5 & 10.5 & 10.5 \\
\hline & 64,7 & 63.6 & 23.7 & 23,7 \\
\hline mittelmäßig & 11,8 & 18,2 & 44,7 & 65.8 \\
\hline schlecht & 3.9 & 3.7 & 15.8 & - \\
\hline sehr schlecht & - & - & 5.3 & - \\
\hline
\end{tabular}

Quelle: Eigene Erhebungen.

\section{Motive und Formen der wirtschaftichen Zusammen- arbeit seit der Grenzöffnung}

Den abweichenden Ausgangssituationen entsprechend differieren auch die vorrangigen Kooperationsmotive. So wird auf ungarischer Seite mit einer Zusammenar- beit vor allem der Transfer von «westlichem» Know-how und Kapital verbunden, der für eine zügige Modernisierung und Umstrukturierung der Betriebe notwendig ist. Auf burgenländischer Seite stehen dagegen die Marktausweitung und die Ausschöpfung komparativer Kostenvorteile in bezug auf das niedrigere Lohn- und Preisniveau in Ungarn im Vordergrund.

Im Hinblick auf die Kooperationsformen ist seit der Grenzöffnung zwar eine deutlich größere Vielfalt festzustellen, dennoch überwiegen nach wie vor die Exportund Importbeziehungen. Daneben treten verstärkt gemeinsame Produktions- und Vertriebsvorhaben sowie Joint-ventures und Arbeitskräftetransfers auf. Direktinvestitionen sind im Hinblick auf die im nördlichen Burgenland vorherrschende kleine und mittlere Betriebsgrößenstruktur dagegen praktisch ohne Bedeutung. Konkret nach den bereits bestehenden oder beabsichtigten grenzüberschreitenden Kooperationen befragt, gaben mehr als die Hälfte der Betriebe in beiden Grenzgebieten an, seit der Ostöffnung bereits grenzüberschreitende Geschäftskontakte geknüpft zu haben oder zumindest diesbezügliche Interessen zu verfolgen. Im direkten Vergleich verfügen die burgenländischen Betriebe über die derzeit intensiveren Aktivitäten (Tab. 2).

Tab.2: Interesse an Geschäftskontakten mit ungarisch/österreichischen Firmen (in \%)

\begin{tabular}{|l|c|c|}
\hline Kontakte & $\begin{array}{c}\text { Interesse osterr. Betriebe } \\
\text { an Kontakten mit } \\
\text { ungar. Firmen }(n=53)\end{array}$ & $\begin{array}{c}\text { Interesse ungar. Betriebe } \\
\text { an Kontakten mit } \\
\text { osterr. Firmen }(n=38)\end{array}$ \\
\hline neu aufbauen & 18.9 & 21.1 \\
erweitern & 28.3 & 28.9 \\
bereits hergestellt & 17.0 & 7.9 \\
konkret geplant & 5,7 & - \\
weder-noch & 30.1 & \\
\hline
\end{tabular}

Quelle: Eigene Erhebungen.

\section{Einflußfaktoren für österreichisch-ungarische Kooperationen}

Art und Umfang der wirtschaftlichen Zusammenarbeit zwischen den Grenzregionen werden wesentlich durch die Betriebsgröße und die Branchenzugehörigkeit bestimmt. Verstärktes Kooperationsinteresse weisen die mittleren und größeren Betriebe sowie die Firmen der Wirtschaftsabteilungen Industrie, Gewerbe und Bauwesen auf. Auch die bereits vor der Ostöffnung aufgebauten Kontakte zu Betrieben im benachbarten Grenzgebiet beeinflussen die grenzüberschreitenden Aktivitäten positiv. Die Art der aus bisherigen Wirtschaftsbeziehungen gewonnenen Erfahrungen wirkt sich kaum auf die Kooperationsbereitschaft aus, was nicht zuletzt auf die grundlegend veränderten Rahmenbedingungen für Geschäftsverbindungen zurückzuführen ist.

Als entscheidende Hindernisse für eine Zusammenarbeit können die Betriebsgrößenunterschiede, die 
Sprachschwierigkeiten und die seitens der burgenländischen Handels- und Fremdenverkehrsbetriebe verbreitete Konkurrenzangst ausgemacht werden. Probleme ergeben sich ferner aus dem rückständigen technologischen Produktionsniveau und den niedrigen Qualitätsstandards auf ungarischer Seite.

Das zukünftige Ausmaß der grenzüberschreitenden Wirtschaftsbeziehungen hängt neben dem allgemeinen Tempo des Transformationsprozesses vor allem davon $a b$, inwieweit die ungarischen Betriebe die sich ihnen bietenden Möglichkeiten umsetzen und die mit der Umstrukturierung verbundenen Krisenerscheinungen meistern können. Auf seiten der burgenländischen Betriebe sind dagegen mehr denn je Flexibilität, Kreativität, Innovationsfähigkeit und Eigeninitiative gefragt, will man die sich aus der Ostöffnung ergebenden Chancen der grenzübergreifenden Kooperation nicht allein den Zentralräumen überlassen.

\section{Gegenwärtige Probleme und zukünftige Entwicklungsmöglichkeiten}

Vor dem Hintergrund der durch die partielle Regionalanalyse und die empirischen Untersuchungen gewonnenen Ergebnisse zu den aktuellen Auswirkungen der Ostöffnung auf die Grenzgebiete sollen im folgenden einige der hieraus resultierenden Entwicklungschancen und -probleme näher betrachtet werden.

\section{Probleme und Chancen im wirtschaftichen Bereich: Arbeitsmarkt und Einzelhandel}

Probleme folgen vor allem aus den zwischen den Grenzgebieten bestehenden einseitigen Grenzgefällen. Dies gilt vor allem für die asymmetrischen Austauschbeziehungen, die insbesondere den burgenländischen Arbeitsmarkt und den Einzelhandel in beiden Grenzgebieten betreffen. Aus den deutlich niedrigeren ungarischen Löhnen, bei einem vergleichbaren Qualifikationsniveau der Arbeitskräfte, ergeben sich sowohl Chancen als auch Probleme. Zwar profitiert die nordburgenländische Wirtschaft von diesem zusätzlichen Arbeitskräftepotential, da durch die Beschäftigung ungarischer Arbeitskräfte der Anteil der Lohnkosten gesenkt, die Wettbewerbsfähigkeit erhöht und der Facharbeitermangel in bestimmten Branchen behoben werden kann. Aus Sicht der Arbeitnehmer verschärft die Beschäftigung von Ungarn jedoch den Lohndruck und führt zu Verdrängungsprozessen innerhalb bestimmter Branchen- und Beschäftigungsgruppen (un- und angelernte sowie ältere Arbeitskräfte). ${ }^{7}$

Eine weitaus größere Gefahr für den burgenländischen Arbeitsmarkt folgt aus der möglichen Verlagerung arbeitskostenorientierter Produktionszweige in das «Niedriglohnland» Ungarn. Der burgenländische Standortvorteil eines unausgeschöpften Arbeitskräftepotentials bei gleichzeitig niedrigen Löhnen ist durch noch vorteilhaftere Bedingungen in Schwellen- und Entwicklungsländern ohnehin nahezu bedeutungslos geworden. Nun erhält das Burgenland zudem Standortkonkurrenz in unmittelbarer räumlicher Nähe.

Auch die häufig propagierte Brückenkopffunktion ${ }^{8}$ erhöht zwar den Status des Burgenlandes innerhalb der internationalen «Standorthierarchie», stellt den regionalen Arbeitsmarkt jedoch vor erhebliche Probleme. Stand das Burgenland bisher als "verlängerte Werkbank» innerhalb der internationalen Arbeitsteilung an letzter Stelle, so wird diese Funktion durch die Peripherisierung der osteuropäischen Ökonomien jetzt Ungarn zugewiesen. Mit fortschreitender politischer und ökonomischer Liberalisierung steigt jedoch die Standortattraktivität Ungarns und sinken entsprechend die burgenländischen Chancen im Wettbewerb um ausländische Direktinvestitionen.

Im Einzelhandel überwiegen auf beiden Seiten der Grenze die Konkurrenzaspekte gegenüber den aus der Grenzöffnung entstandenen Möglichkeiten der Zusammenarbeit. Nach dem jahrelangen einseitigen Kaufkraftabfluß durch den burgenländischen Einkaufstourismus in Ungarn setzte mit der Reisefreiheit der ungarischen Bevölkerung ein Boom im burgenländischen Einzelhandel ein, der jedoch erwartungsgemäß nicht anhielt. Neben einer gewissen Sättigung des Nachholbedarfs an Konsumgütern bestimmt vorerst die durch steigende Arbeitslosigkeit und sinkende Realeinkommen begrenzte Kaufkraft der ungarischen Bürger das Ausmaß des grenzüberschreitenden Einkaufsverkehrs. Darüber hinaus sind die positiven Auswirkungen des grenzübergreifenden Einkaufstourismus aus der Sicht des burgenländischen Einzelhandels eher gering, da durch die Richtung des Preis- und Kaufkraftgefälles der burgenländische Kaufkraftabfluß nach Ungarn höher ausfällt als die ungarische Nachfrage im Burgenland. Letztere wird zudem, auch bedingt durch die geringe Attraktivität und Konkurrenzfähigkeit des burgenländischen Einzelhandels, zum Großteil außerhalb des Burgenlandes gedeckt. Kurz- bis mittelfristig sind im nördlichen Burgenland positive Effekte daher vor allem im kleinräumigen Nachbarschaftshandel zu erwarten.

Für die grenznahen und höherrangigen westungarischen Zentren bestehen durch die Grenzöffnung Möglichkeiten, dauerhaft Versorgungsfunktionen für Teile der nordburgenländischen Bevölkerung zu übernehmen. Besonders für Sopron sind die Chancen, die früheren Einzugsgebiete auf burgenländischer Seite zurückzugewinnen, relativ groß. Zu den negativen Begleiterscheinungen des grenzüberschreitenden Einkaufsverkehrs zählen die Veränderungen des Preisniveaus (Preisverfall auf österreichischer, Preisanstiege auf ungarischer Seite) und entsprechende Absatzschwierigkeiten. 


\section{Chancen und Probleme im soziokulturellen Bereich}

Die langfristig wohl größten Chancen beiderseits der Grenze existieren im soziokulturellen Bereich. Hier kann zudem auf langjährige Kontakte zurückgegriffen werden, die seit Mitte der 70er Jahre mit dem allmählichen Abbau des Grenzzonenstatus und der Abriegelung der Grenze wieder aufgenommen und intensiviert wurden. Seither bestehen vor allem auf den Gebieten Sport, Kultur und Fremdenverkehr grenzüberschreitende Beziehungen. Der Fall des Eisernen Vorhangs eröffnet nun die Möglichkeit, auch die nachbarschaftlichen Kontakte wiederzubeleben. Voraussetzung hierfür ist die Errichtung (weiterer) kleinräumig wirksamer Grenzübergänge, da die jenseits der Grenze gelegenen Nachbardörfer bislang häufig nur über erhebliche Umwege erreicht werden können. Die Errichtung neuer Grenzstellen löst jedoch zunehmend kontroverse Diskussionen innerhalb der burgenländischen Grenzbevölkerung aus. Die gegen die Öffnung weiterer Übergänge angeführten Gründe reichen von Umwelt- und wirtschaftlichen Aspekten bis hin zu Vorbehalten gegenüber den «Fremden». Um die bestehenden Chancen nutzen zu können, stellt der Abbau der «Grenze im Kopf» eine vordringliche Aufgabe dar. Ein wesentliches Hemmnis diesbezüglich sind die mangelnden Sprachkenyntnisse. ${ }^{9}$

\section{Tatsächlicher Grad der Durchlässigkeit}

Die genannten asymmetrischen Austauschbeziehungen haben staatlicherseits zu einseitigen Einschränkungen der Durchlässigkeit der Staatsgrenze geführt. So wurden beiderseits strengere Grenzkontrollen eingeführt, die Zahl der ausländischen Arbeitskräfte im Burgenland durch das Ausländerbeschäftigungsgesetz begrenzt und die zoll- und devisenrechtlichen Bestimmungen auf ungarischer Seite verschärft. Von einer «offenen Grenze» im österreichisch-ungarischen Grenzraum kann gegenwärtig daher nicht gesprochen werden.

\section{Entwicklungschancen und -probleme im direkten Vergleich beider Grenzgebiete}

Aufgrund der ausgewogeneren Wirtschafts- und Siedlungsstruktur werden die aus der Grenzöffnung resultierenden Entwicklungschancen mittel- bis langfristig für das westungarische Grenzgebiet größer sein als für das nördliche Burgenland. Voraussetzung ist, daß der Transformationsproze $\beta$ in Ungarn weiter voranschreitet und da $\beta$ die Anpassungsrezession überwunden werden kann. Aus burgenländischer Sicht zeigt sich, daß der peripherieräumliche Charakter der Wirtschaft durch die Grenzöffnung nicht entscheidend verändert wird. Vielmehr ist mit zusätzlichen Funktionsverlusten infolge neuer Konkurrenzverhältnisse zu rechnen. ${ }^{10}$

In jedem Fall erfordert die Ausschöpfung der bestehenden Chancen und die Minimierung der Probleme flexibles und rasches Handeln seitens der staatlichen und privaten Akteure sowie eine verstärkte grenzübergrei- fende Zusammenarbeit. Die eigentlichen Vorteile für die Grenzgebiete dürften gerade in den kleinräumigen grenzüberschreitenden Kooperationsformen liegen.

\section{Ausblick: Perspektiven der Grenzregionen im Rahmen der gesamteuropäischen Neuordnung}

Über die politisch-ökonomischen und gesellschaftlichen Veränderungen in Osteuropa hinaus wird die Entwicklung des österreichisch-ungarischen Grenzraumes schließlich auch wesentlich durch die Öffnung zur Europäischen Union (EU) bestimmt. So zieht die Assoziation Ungarns und der Beitritt Österreichs zur EU eine weitere Veränderung der Wertigkeit der Staatsgrenze zwischen beiden Ländern und damit auch der Rahmenbedingungen für die Grenzgebiete und die grenzüberschreitenden Beziehungen nach sich. Seit dem Zweiten Weltkrieg bis heute hat sich die österreichisch-ungarische Grenze somit von einer Systemgrenze zwischen Kapitalismus, Demokratie und Marktwirtschaft einerseits sowie Sozialismus, Diktatur und Zentralverwaltungswirtschaft andererseits zur EU-Außengrenze gewandelt. Mit einem Beitritt Ungarns zur Union würde sie als EU-Binnengrenze weiter an Bedeutung verlieren. Gleichzeitig nimmt der Grad der Durchlässigkeit und damit der verbindenden Funktion der Grenze weiter zu. Die parallel zum Funktionswandel der Staatsgrenze erfolgende Umbewertung der Grenzgebiete wird besonders im ungarischen Untersuchungsgebiet deutlich. Einer qualitativen Abwertung des Grenzgebietes und der Behinderung der wirtschaftlichen Entwicklung nach dem Zweiten Weltkrieg, folgt(e) nach dem Fall des Eisernen Vorhangs die Bevorzugung der Grenzgebiete infolge der günstigen Lage zu Westeuropa.

Insgesamt kann die österreichisch-ungarische Grenze somit als ein klassisches Beispiel für die Funktionsveränderung von Grenzen und die Abhängigkeit ihrer Wertigkeit von den gesellschaftlichen und politisch-wirtschaftlichen Systemen der aneinandergrenzenden Staaten sowie deren Zugehörigkeit zu übernationalen Gemeinschaften und Organisationen angesehen werden.

\section{Anmerkungen}

${ }^{1}$ Vgl. BRÜCHER, W. (1989): Einführung. In: SOYEZ, D., u. a. (Hrsg.): Das Saarland, Bd. 1: Beharrung und Wandel in einem peripheren Grenzraum, Saarbrücken, S.125.

2 Vgl. z.B. VON MALCHUS, V. (1975): Partnerschaft an europäischen Grenzen. Integration durch grenzüberschreitende Zusammenarbeit, Bonn (= Europäische Schriften des Instituts für Europäische Politik 39/40), und die dort angegebene Literatur. 
3 Dies belegen die seither erschienenen zahlreichen Aufsatzsammlungen wie z. B. MANDL, P., WASTL-WALTER, D. (Hrsg.) (1992): Regionalforschung von grenzüberschreitender Bedeutung in Mitteleuropa, Wien (= AMR Info 23); SEGER, M., BELUSZKY, P. (Hrsg.) (1993): Bruchlinie Eiserner Vorhang. Regionalentwicklung im österreichisch-ungarischen Grenzraum, Wien (= Studien zur Politik und Verwaltung 42); SCHAMP, E. (Hrsg.) (1995): Neue grenzüberschreitende Regionen im östlichen Mitteleuropa, Frankfurt (= Frankfurter Wirtschafts- und Sozialgeographische Schriften 67); oder BÜRKNER, H.-J., KOWALKE, H. (Hrsg.) (1996): Geographische Grenzraumforschung im Wandel, Potsdam, (= Praxis Kultur- und Sozialgeographie 15).

${ }^{4}$ Von den umfangreichen Einzelergebnissen können hier nur einige exemplarisch in Form von Graphiken und Tabellen wiedergegeben werden.

5 Als Durchschnittswert wurde für die verschiedenen haushaltsspezifischen Merkmale der sogenannte Häufigkeitsin$\operatorname{dex} H_{i}$ berechnet. Den acht Häufigkeitsstufen entsprechend wurden dazu mit steigender Häufigkeit des Grenzübertritts Punkte von null (nie) bis sieben (mehrmals pro Woche) vergeben und anschließend das arithmetische Mittel, d. h. der Quotient aus der Summe der Nennungen je Stufe, multipli- ziert mit der entsprechenden Punktzahl und der Gesamtzahl der Antworten je Merkmal, bestimmt.

${ }^{6}$ Zur grenzüberschreitenden betrieblichen Zusammenarbeit vgl. auch die folgenden empirischen Studien: IFES (1990): Expansionschancen in grenznahen Regionen, Wien, sowie ÖIBF (Hrsg.) (1990): Wirtschafts- und Qualifikationsentwicklung der Grenzregionen in Österreich und Ungarn, Wien, 4 Bde.

${ }^{7}$ Vgl. auch JANDRISITS, W. (1991): Ostöffnung. Burgenlands Chance oder...? In: DEINHOFER, E., HORVATH, T. (Hrsg.): Grenzfall. Burgenland 1921-1991, Eisenstadt, S. $304 \mathrm{ff}$.

${ }^{8}$ Vgl. z. B. SIPÖTZ, H. (1990): Aspekte der zukünftigen Landesentwicklungspolitik. In: FASSMANN, H., PRÖLL, U. (Hrsg.): Standort Burgenland. Probleme und Entwicklungschancen der Peripherie, Eisenstadt, S. $96 \mathrm{ff}$.

9 Vgl. ÖIR (1991): Szenarien zur Ost-Grenzöffnung und deren Auswirkungen auf die österreichischen Ost-Grenzregionen, Wien, S. 43 und S. 50.

${ }^{10} \mathrm{VgI}$. JANDRISITS, W. (1991), a. a. O., S. 309, und SCHNEIDEWIND, P. (1991): Neue Chancen für das Grenzland. In: ÖROK (Hrsg.): Offene Grenzen - Neue Aufgaben für die Regionalpolitik, ÖROK-Enquete, Wien 1991, S. 55-60 (= ÖROK-Schriftenreihe 94). 